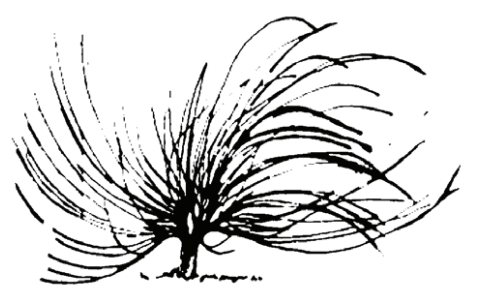

\title{
Experiencias de Aula -y su Proyección en la Dinámica de Trabajo de Campo- en la Formación Docente de la Carrera de la Enseñanza del Español
}

\author{
Marlene Aguirre Chaves ${ }^{1}$ \\ Universidad Nacional \\ Heredia, Costa Rica \\ maguirrech@hotmail.com
}

\begin{abstract}
Resumen
El planteamiento de este ensayo tiene como objetivo primordial, exponer las experiencias de un trabajo realizado en forma simultánea, -de aula y de campo-, de manera sostenida, en los cursos de la carrera de Bachillerato de la Enseñanza del Español y que se acentúa en los dos últimos cursos: DEY458 Desafíos Didácticos en la Práctica Docente y LCG400 Seminario de Innovación y Producción Educativa, los cuales podrían tener el calificativo, dependiendo de su contextualización, de un trabajo de extensión o investigación. Se presentan algunas experiencias del trabajo desarrollado, mediante cuatro factores que intervienen en ese proceso: los escenarios en los cuales se ubican los estudios de investigación; la mediación pedagógica, que es un espacio de interacción entre
\end{abstract}

(c) (1) $(\theta)$

Recibido: 15 de febrero de 2015-Aprobado: 13 de abril de 2015

1 Licenciada en Ciencias de la Educación con Énfasis en la Didáctica de Español. Académica-investigadora en la División de Educología-CIDE, Universidad Nacional. Ha impartido cursos en la Carrera de Bachillerato en la Enseñanza de Español como pedagoga. Directora de la Revista Ensayos Pedagógicos de la División de Educología, CIDE, Universidad Nacional. Profesora de Español en el Ministerio de Educación Pública de Costa Rica. 
los elementos del proceso de la enseñanza y aprendizaje y actores involucrados; la formación docente que tiene una participación activa en todo el quehacer académico; por último, el modus vivendi como un factor de cambio, marcado por la era de las tecnologías de la información y el fenómeno de la transculturación.

Palabras clave: experiencias de aula, trabajo de campo, mediación pedagógica, enseñanza del Español, formación docente, escenarios, modus vivendi

\begin{abstract}
This essay has as its main objective to describe the experiences of the fieldwork and classwork done simultaneously and steadily in the courses for the Bachelor's Program for Spanish Teaching at "Universidad Nacional," Costa Rica, which are stressed in the last two courses "DEY458 Desafíos Didácticos en la Práctica Docente" and "LCG400 Seminario de Innovación y Producción Educativa" and which may be described, depending on their conceptualization, as research or outreach projects. Different experiences about the work done are presented by referring to four factors that play a role in this process, the scenarios in which the research projects are located, pedagogical mediation, which is a space for interaction between the elements of the teaching-learning process and the participants involved, teacher training, which plays an active role in any academic activity, and lastly, the modus vivendi as a change factor influenced by the era of information technologies.
\end{abstract}

Keywords: classroom experiences, fieldwork, pedagogical mediation, Spanish teaching, teacher training, scenarios, modus videndi

\title{
Consideraciones iniciales
}

Los cursos de la carrera de Bachillerato en la Enseñanza del Español, de la División de Educología, de la Universidad Nacional, se caracterizan por incluir en sus programas, la investigación de manera paulatina, de tal forma, que los alumnos desde sus cursos iniciales dan 
sus primeros pasos en una investigación de campo pero sostenida a lo largo de su carrera.

Es así como, desde los cursos iniciales se establece un vínculo permanente entre el espacio universitario y las instituciones educativas que los mismos estudiantes han elegido para trabajar en los diferentes cursos, lo cual ha sido muy provechoso porque permite hacer un trabajo contextualizado.

De esta manera, los estudiantes se informan sobre las distintas modalidades académicas que ofrece el Ministerio de Educación Pública, entre ellas se pueden mencionar: los colegios académicos diurnos, nocturnos, técnicos vocacionales, científicos y otros; los cuales, históricamente han formado parte de manera voluntaria en la formación de los futuros profesionales, por lo tanto, los estudiantes de esta carrera, han tenido experiencias diferentes tanto a nivel académico, cultural, de costumbres y valores de las comunidades y contextos en que geográficamente las instituciones educativas de la secundaria costarricense están ubicadas. Los estudiantes de esta carrera se nutren de los conocimientos y experiencias de los profesores en servicio y del entorno en los cuales participan.

Estas experiencias que los alumnos han vivenciado se traen a las aulas universitarias y son compartidas a los demás compañeros del grupo, estableciendo un nexo que les permite hacer un intercambio en distintos sentidos:

1. Escenarios

2. Mediación pedagógica

3. Formación docente

4. Modus vivendi académico

\section{Escenarios}

Es interesante cuando se comparten las vivencias de otros escenarios, que quizás solo el profesor universitario pueda conocer mediante las visitas que registran los alumnos, cada vez que van al campo a realizar sus investigaciones. Este acercamiento o contacto puede darse de dos formas: ya sea porque el alumno es originario de esa comunidad, o bien, por otros intereses de cercanía o de afinidad. Cabe resaltar que en los últimos años, el Proyecto "Colegios Amigos", de la División de Educología, de la Universidad Nacional, ha venido consolidando un trabajo con algunas 
instituciones educativas de la secundaria pública costarricense, para que estas no solo le brinden el espacio académico a los estudiantes que van a realizar su práctica docente, sino que desarrollen acciones de trabajo conjuntas con los profesores de esas instituciones educativas. En este momento, el Proyecto "Colegios Amigos" se ha convertido en un aliado y apoyo tanto para los profesores que dan el curso DEY458 Desafíos Didácticos en la Práctica Docente como para que sus estudiantes puedan sentirse más respaldados y tranquilos, existiendo un convenio entre las partes que les proporciona estabilidad y seguimiento.

Sin duda, estos enlaces, nos han permitido fortalecer vínculos, entre las instituciones educativas de la secundaria costarricense y la Universidad Nacional, fortalecer aspectos teóricos, costumbres y valores de las comunidades, conocer los recursos y materiales disponibles que brindan a la población estudiantil, la infraestructura, el personal académico y administrativo con que se cuenta, relación con los padres de familia y el apoyo de la comunidad vinculada con las actividades que desarrolla la institución educativa.

\section{Mediación pedagógica}

El estudiante próximo a concluir sus estudios de Bachillerato en la Enseñanza de Español debe tener amplio conocimiento y dominio sobre las situaciones de enseñanza y aprendizaje que interactúan en el marco de la mediación pedagógica. La experiencia sostenida a lo largo de los años de la carrera, le permite poseer una visión más amplia sobre metodología, técnicas, estrategias, evaluación, manejo de equipo y recursos tradicionales y tecnológicos, dominio de contenidos del programa de estudios de la disciplina vigente y manejo de grupos con amplia visión sobre aspectos de género, etnia, religión, etc., en forma contextualizada, es decir, vinculada a las realidades de los contextos educativos.

Según Álvarez del Valle (2004), la función del mediador es “cuestionar las posturas de las partes para hacerles ver la luz. En ese sentido, la mediación se transforma en pedagógica, cuando el procedimiento por el cual "el mediador" -uno de los tres integrantes de la tríada: docente, alumno, contenido, logra facilitar la resolución del conflicto cognitivo entre las otras dos partes intervinientes. La función del mediador es cuestionar la postura de las partes para lograr la apropiación del conocimiento", págs. 18-21. 
La afirmación anterior, se despeja en la serie de acciones voluntarias que van surgiendo entre los distintos actores, por ello, el campo de la mediación pedagógica, quizás sea uno de los más enriquecedores y complejos, por cuanto presenta un híbrido, pues se conjuga la disciplina con el saber pedagógico de manera integral y en estrecha interacción con los elementos del proceso de enseñanza y aprendizaje, y esto, tiene el mérito de la formación del profesorado en estas dos líneas (trabajo de aula y de campo), que he venido planteando.

La mediación pedagógica es un proceso determinante, ${ }^{2 *}$ como el motor que viene a impulsar todo lo demás", si queremos formar excelentes profesionales, en el seno de una buena práctica de mediación pedagógica, está el secreto. La cordialidad, la forma de mediar y desarrollar los contenidos, la cautela, el aprender a escuchar, el respeto, el saber seleccionar los materiales y los recursos requeridos, la empatía, la motivación, el placer y el gusto por enseñar y una buena planificación y preparación son elementos indispensables para lograr una buena formación. He dejado, el componente de la evaluación, para tratarlo por aparte, porque quizá sea uno de los elementos de la mediación, más delicados de trabajar, una mala aplicación de este puede ocasionar una serie de trastornos personales, los cuales pueden ser decisivos para tomar decisiones anticipadas que van a incidir en el proyecto de vida de un estudiante. Así nos encontramos con anécdotas de estudiantes como las siguientes: "me quedé por medio punto", "nunca vimos esa materia en clase", "el examen era diferente a las prácticas", "me estudié todo y no salió nada", "el examen estaba muy enredado"; en fin, es interminable la lista de comentarios y justificaciones que escuchamos al término de una prueba. Este tipo de argumentos han sido vivenciados por los estudiantes de la secundaria costarricense, y ahora como estudiantes de la enseñanza del español, y cuando se discuten en el seno de la clase, les sirve para comparar experiencias en estos dos contextos, lo cual le va ayudar al futuro profesional a poseer más herramientas para mejorar los procesos de una evaluación más contextualizada y más auténtica.

El educador debe tener claridad de que la evaluación es un proceso, en el que también intervienen una serie de factores concebidos en el marco de una relación entre personas con características muy disímiles, $\mathrm{y}$ en estas relaciones, el objeto de fondo, debería ser el conocimiento

2 Término empleado por la autora de este ensayo. 
mediado, donde se manifiesta el interés y la dificultad de unos más que en otros, produciendo un desfase que limita o favorece el proceso. Ahora bien, esta brecha que comienza a gestarse si no se atiende de manera oportuna, se concreta en debilidad cuando estos conocimientos sean acreditados. Entonces, la evaluación como tal, tiene implicaciones no solo cuando "algo" debe ser resuelto, sino su debilidad empieza en el proceso de la mediación pedagógica porque ese "algo" no fue comprendido de la mejor manera. Así, se podría desarrollar un contenido tomando en cuenta distintas habilidades que le permitan la comprensión, el análisis, la aplicación o la síntesis; cualquier nivel de conocimiento en el que no se hayan logrado los objetivos, estaría en desventaja para el mediado. Por otra parte, si estas capacidades no se han trabajado adecuadamente en la mediación pedagógica, difícilmente los resultados de la evaluación serán positivos.

La evaluación debe entenderse como un proceso integral de carácter formativo, que sirva para fortalecer y retroalimentar las prácticas educativas, y que no sea vista como una sanción, un castigo, un obstáculo para crecer como ser humano y profesional.

\section{Formación docente}

Sin duda, este es un tema polémico en la actualidad. Las razones podrían ser muy variadas, una de ellas es la influencia que ha tenido la tecnología en los procesos de la enseñanza y aprendizaje. En algunos casos, los especialistas en materia educativa y pedagogos señalan la poca formación docente para desarrollar ambientes tecnológicos, en otros, la falta de actualización de los docentes y de manera más debatida, la formación del profesorado universitario; en estos pilares se sostienen algunas debilidades que se manifiestan en la mediación pedagógica, por citar algunas: adecuaciones curriculares, diversidad cultural, manejo de grupos, transculturalización, metodologías, evaluación y otros.

Por su parte, hoy los jóvenes han cambiado su manera de pensar. Lo que hace algún tiempo era prohibido, o era parte de un tabú, ahora para ellos es normal, esta situación puede darse en el ámbito de las relaciones entre pares, en sus modales, costumbres, en sus valores y actitudes. Definitivamente, estos cambios producen enfrentamientos de tipo generacional y obstaculizan las buenas relaciones entre educador y educando, creando un distanciamiento que en la mayoría de los casos 
no termina dando buenos frutos. Podría decirse que en el tema de formación docente hay mucha "tela que cortar", los tiempos han cambiado y la incertidumbre por aspirar a formar a una persona con los mejores valores e ideales de buen ciudadano, cada vez se torna más complejo.

Por otra parte, "el buen maestro", aquel que con sus palabras inspira y guía al discípulo, que el motivo de enseñar, el gusto y el amor como sus principales fuentes auténticas de mediar procesos ya no son tan relevantes, y en su lugar, impera un desamor para el que aprende. Hay que mirar todos los alcances de este "entretejer", por un lado, quienes somos responsables directos -formación docente universitaria-, responsables indirectos -educadores de secundaria en servicio-, responsables en proceso - estudiantes en formación. Este complejo accionar se desarrolla en un marco político, social y cultural de nuestra sociedad costarricense.

\section{Modus vivendi académico}

La forma en que las personas organizan sus vidas académicas ha variado, así los alumnos toman decisiones basadas en la práctica, en su cotidianidad, en sus vivencias personales y no se interesan tanto en la teoría, en la lectura de extensas obras, en la concentración por largas horas, en el esfuerzo mental, en lo estático; prefieren lo práctico, la combinación entre lo recreativo y el estudio, la socialización del conocimiento, la observación, la aplicación de lo que han aprendido de sus precursores.

Este modus vivendi ha marcado una nueva ruta en la manera de concebir los procesos de la enseñanza y aprendizaje, de ahí que, constantemente hay un resentimiento académico por parte de los docentes que atribuyen estos cambios a la falta de valores y actitudes de los jóvenes: la presentación personal, peinados, formas de saludar, relaciones con sus compañeros, los tonos de voz un tanto despectivos, la concepción y administración del tiempo, los valores y principios religiosos, los encuentros amorosos, las relaciones de pareja, la actitud ante la vida (rompen patrones familiares, culturales, sociales, políticos). Una apreciación similar se presenta en esta afirmación, que hace Rodríguez (2006),

... los adultos han ido perdiendo el principio de autoridad y otros valores que sustentaban el viejo orden tradicional. Se produce un fuerte rechazo del pasado y adquiere valor todo lo nuevo, todo lo que suena a moderno, a vanguardia. Si hubo un tiempo en que la 
gente miraba a sus mayores como modelo a seguir en su forma de vida, sus modales, su lenguaje, a partir de ahora el mimetismo cambia su dirección y son los mayores los que imitan y pretenden parecerse a los jóvenes. (p. 7)

Ahora los jóvenes tienen otras preferencias, por ejemplo, disfrutar de una película en casa, de un juego, de la utilización de redes sociales (Facebook, Twitter, etc.), los avances tecnológicos han hecho que estos recurran a otros mecanismos para emplear su tiempo libre. Las innovaciones tecnológicas y el desarrollo de las redes sociales han creado una comunicación constante entre sus pares mediante mensajes de texto, Whats App, Viber, Line, correo electrónico; utilizan buscadores de información, galería de fotos, presentaciones y otras aplicaciones que pueden disfrutar en su teléfono móvil o "tablets", el avance tecnológico ha revolucionado el modus vivendi de los jóvenes, de allí que es necesario que incorporemos las TIC's, (tecnología de la información y comunicación), en el trabajo de mediación pedagógica. La incorporación es necesaria y urgente, los alumnos requieren clases más dinámicas e interactivas, sentirse más cercanos a sus vivencias, sentires y percepciones, con mayor libertad para crear e innovar, al mismo tiempo, reflexionando sobre su quehacer.

En estos cuatro grandes componentes que he citado, he visualizado el trabajo de investigación que se coordina desde las aulas universitarias con las instituciones educativas del país, el cual se ha trabajado en cada uno de los cursos de la carrera de Bachillerato en la Enseñanza de Español, no obstante, este esfuerzo se concreta en los cursos DEY458 Desafíos Didácticos en la Práctica Docente y LCG400 el Seminario de Innovación y Producción Educativa del Español en Costa Rica, que son los dos cursos finales de la formación de bachillerato en la Enseñanza del Español, en los cuales, los alumnos tienen la oportunidad de vivenciar y aplicar los frutos de toda su carrera, al mismo tiempo, se logra consolidar el trabajo de campo, de manera sostenida y vinculada con las partes. Los estudiantes de estos cursos han llevado un hilo conductual que les permite estar preparados para enfrentarse a los desafíos actuales.

Así el curso DEY458 Desafíos Didácticos en la Práctica Docente, está planteado en dos grandes vías: las sesiones presenciales en la Universidad y la permanencia en una institución educativa de secundaria, donde los estudiantes tienen la posibilidad de desarrollar su 
práctica durante un período, a la vez, inician un proceso de observación relacionado con las realidades de los contextos, modalidades, procesos de aula, teoría versus práctica, abordajes metodológicos y otras situaciones relacionadas con los elementos que interactúan en el proceso de la enseñanza y aprendizaje. A partir de este momento, los alumnos se incorporan a un proceso comparativo con lo enseñado en las aulas universitarias y las realidades de los contextos educativos, se inicia la separación de sus profesores, quienes han sido sus tutores y modeladores, y comienzan a trazar el suyo propio. En esa formación de identidad profesional, se inicia también la reflexión sobre su misma práctica, aquí es cuando el alumno empieza a plantearse algunas interrogantes: ¿Qué tipo de docente quiero ser?, ¿me siento preparado para enfrentar los desafíos actuales?, ¿cómo puedo abordar mi práctica en forma eficiente?, ¿es significativo lo que hago?, ¿cuáles son mis alcances?, y otros, que conforme se desarrolla el proceso, el estudiante va aclarando.

En esta nueva etapa, surge la inseguridad, la angustia, los estados emocionales, el arrepentimiento, la desesperación, el llanto, la desmotivación, la intolerancia y el miedo como diría Paulo Freire (1970), "asumir el miedo no es esconderlo, es analizar su razón de ser" la felicidad, la alegría, la satisfacción, el orgullo, la empatía son los menos, p74.

En este marco de acciones, el estudiante inicia su trayectoria como profesional y se da el engranaje con el siguiente curso, LCG400 el Seminario de Innovación y Producción Educativa del Español en Costa Rica. Su objetivo principal es procurar que el estudiantado lleve a cabo estudios sistemáticos e investigaciones sobre la enseñanza del español, mediante propuestas metodológicas y prácticas para el estudio del español hablado en Costa Rica. Se hace hincapié en el análisis de casos, con el fin de que se pongan en práctica teorías, aproximaciones metodológicas y toda la fundamentación propia de una investigación académica. Este curso es la base para desarrollar procesos de índole investigativa, que más adelante puedan servir para que el alumno continúe sus investigaciones en los cursos de licenciatura.

Actualmente, este curso es colegiado con la Escuela de Literatura y Ciencias del Lenguaje y es impartido por dos académicas, una de la División de Educología y otra de la Escuela de Literatura.

Se debe destacar que el curso en esta modalidad, ha dado muy buenos frutos, los alumnos tienen la oportunidad de integrar los saberes pedagógicos, los de la disciplina de español y la puesta en marcha de los 
contenidos de los programas de español que se aplican en las instituciones de educación secundaria en nuestro país. Este trabajo,en tres direcciones, permite el desarrollo de la investigación-acción, la cual surge desde los diferentes escenarios y tiene como característica que sus aportes sirvan para fortalecer el trabajo desarrollado en las aulas, para la partida de otras investigaciones, para dar a conocer el trabajo que realizan los académicos de la División de Educología con sus alumnos, de manera interdisciplinaria e intercambiar estas experiencias con la comunidad universitaria y del CIDE.

A manera de ejemplo, voy a citar un trabajo de investigación, en el cual formé parte como académica del curso LCG400 Seminario de Investigación y Producción Educativa y en el que participaron dos estudiantes de este curso (Arguedas y Ureña, 2010), estas dos jóvenes proceden de zonas rurales y curiosamente a raíz de sus vivencias personales, y como especialistas en la disciplina de Español, se interesaron por investigar ${ }^{3 " L a}$ influencia que ejerce la utilización de regionalismos en el inventario léxico oral de los estudiantes de las secciones 7-3 y 8-1 del Liceo El Roble, Heredia".

El planteamiento de este estudio tiene antecedentes interesantes que surgen a partir de una vivencia personal de las investigadoras, primero como estudiantes de la secundaria, luego, como estudiosas de la lingüística. Al realizar su práctica docente, se interesaron por identificar la metodología que utilizaban los profesores para enseñar el tema de regionalismos. Las investigadoras estaban conscientes de que el propósito del estudio era fomentar la utilización de un léxico amplio y adecuado en el nivel oral de los alumnos, a la vez, dejar de lado la idea de la normativa de la lengua y demostrar que la función educativa no radica en promover un lenguaje ideal, sino más bien en rescatar la riqueza léxica que poseen los estudiantes de acuerdo con su contexto, enseñar al estudiante a adecuar su vocabulario de acuerdo con la situación comunicativa antes que minimizar su léxico e imponerle el lenguaje culto, desconocido para él y lejano a su realidad lingüística.

En importante destacar que los conocimientos previos de las investigadoras: el paso por la secundaria, luego, la pertenencia a una comunidad rural y posteriormente como estudiosas de la lengua; les permitió desarrollar una investigación científica rigurosa y seria.

3 Tema de investigación abordado por Arguedas y Ureña, estudiantes de la carrera de Bachillerato en la Enseñanza de Español en el 2010. 


\section{Principales conclusiones y hallazgos}

La experiencia académica que adquieren los docentes y sus estudiantes, en los cursos DEY458 Desafíos Didácticos en la Práctica Docente y LCG400 el Seminario de Innovación y Producción Educativa del Español en Costa Rica, refleja un aporte valioso en la formación de los futuros profesionales de la Enseñanza del Español, por esta razón me permito mencionar algunas consideraciones finales.

a. Es necesario que los estudiantes en formación estén vinculados durante toda su carrera con los contextos educativos de manera permanente, dado que esto les permite poseer mayores herramientas metodológicas y una mejor comprensión de las realidades educativas.

b. El curso de DEY458 Desafíos Didácticos en la Práctica Docente permite que los estudiantes apliquen los conocimientos adquiridos durante toda su carrera, tanto de la disciplina como pedagógicos, a la vez, relacionar la teoría con la práctica, crear y ejecutar nuevas estrategias para la mediación pedagógica, desarrollar su propia identidad profesional, determinar carencias y debilidades en el proceso de enseñanza y aprendizaje, seleccionar los recursos más óptimos, apropiarse del dominio de los contenidos de la disciplina, fortalecer y proponer alternativas para mejorar su propia práctica, permitiendo una contribución significativa para mejorar y fortalecer los procesos de la enseñanza y aprendizaje.

c. El curso LCG400 Seminario de Innovación y Producción Educativa del Español en Costa Rica, desarrolla un trabajo de carácter interdisciplinario, (los saberes pedagógicos y el saber disciplinario), ambos componentes forman parte de la carrera de la Enseñanza de Español y es un curso colegiado. Se desarrolla un trabajo en forma conjunta e integrada, en equipo, permitiendo la realimentación del saber pedagógico y disciplinario, la dinámica de relación de los saberes y su aplicación. Además, se generan vínculos académicos que dan "pie" a futuras investigaciones. En ambos cursos, se hace una revisión bibliográfica, permitiendo la actualización de conocimientos tanto del saber pedagógico como disciplinar. El alumno adquiere experiencia en el manejo y sistematización de la información, aprende a elaborar instrumentos, 
revisa teorías y elige su pertinencia, selecciona las mejores técnicas para recabar información, manipula datos, distingue las partes de una investigación cualitativa y aprende a redactar un informe de investigación. El alumno adquiere destrezas y habilidades para organizar información, analizarla y sintetizarla y socializar los hallazgos más relevantes en un determinado escenario. Los alumnos del curso de Seminario de Innovación y Producción Educativa, del Español de Costa Rica, obtienen las herramientas básicas para desarrollar o continuar una investigación a nivel de licenciatura.

i. Cabe destacar, que la experiencia de Arguedas y Ureña (2010), se socializó en el salón de clase y posteriormente se presentó como ponencia en el tercer FIPED realizado en el 2010, en nuestro país. Véase, en apéndice una muestra de regionalismos, utilizados por alumnos de la comunidad del Liceo del Roble, Heredia.

j. Otra experiencia valiosa, es la posibilidad que tienen los estudiantes de presentar un artículo como producto de su investigación y publicarlo en la Revista Ensayos Pedagógicos, la cual ofrece un espacio para que los estudiantes de las carreras de bachillerato en la enseñanza de las distintas disciplinas, que ofrece la División de Educología conjuntamente con las escuelas, puedan divulgar los resultados de su investigación. Así, han participado estudiantes de Estudios Sociales, Ciencias, de Español, las estudiantes Michelle Arteaga Guerrero y Rebeca Cortés Esquivel, con el artículo "'Producción de Materiales Didácticos en el Tema de Sintaxis del Español para Personas con Retinosis Pigmentaria y Ceguera Total", el cual recomienda algunos materiales especializados para enseñar a los estudiantes de secundaria con discapacidad visual.

\section{Referencias bibliográficas}

Alvarez del Valle, E. (2004). "XII Jornadas de Reflexión Académica en Diseño y Comunicación: Procesos y Productos. Experiencias Pedagógicas en Diseño y Comunicación" Año V, Vol. 5, Buenos

4 Revista Ensayos Pedagógicos. División de Educología, Centro de Investigación y Docencia en Educación, Universidad Nacional. Volumen 8 no.2, julio-diciembre-2013. 
Aires, Argentina, págs.18-21. Recuperado de http://fido.palermo.edu/servicios_dyc/publicacionesdc/vista/detalle_articulo. php?id_libro $=120 \&$ id_articulo $=447$

Avedaño, F., Miretti, M.L. (2006). El desarrollo de la lengua oral en el aula: Estrategias para enseñar a escuchar y hablar. Argentina: Homo Sapiens Ediciones.

Arguedas y Ureña (2010). La influencia que ejerce la utilización de regionalismos en el inventario léxico oral de los estudiantes de las secciones 7-3 y 8-1 del Liceo El Roble, Heredia”. (Trabajo de investigación desarrollado en el curso de Seminario de Innovación y Producción Educativa, del Español en Costa Rica. División de Educología-Escuela de Literatura y Ciencias del Lenguaje), Universidad Nacional, Costa Rica.

Arteaga Guerrero, M. y Cortés Esquivel, R. (2013, en prensa). Producción de Materiales Didácticos en el Tema de Sintaxis del Español para Personas con Retinosis Pigmentaria y Ceguera Total. Ensayos Pedagógicos, VIII, (2).

Freire, Paulo, (2004). Cartas a quien pretende enseñar- 1ra ed. 2a reimp.- Buenos Aires: Siglo XXI Editores Argentina. Recuperado de http://baseddp.mec.gub.uy/Documentos/Bibliodigi/cartas $\% 20 \mathrm{a} \% 20$ quien $\% 20$ preten de $\% 20$ ensenar.pdf.

Lomas, C. (1999). Cómo enseñar a hacer cosas con las palabras. Teoría y práctica de la educación lingüistica. Volúmenes I y II. España: Edit. Paidós.

Rodríguez, F. (2006). Medios de comunicación y contracultura juvenil. Revista de Lingüística aplicada a la comunicación. Circulo. Universidad Complutense de Madrid, España. Recuperado de http:// pendientedemigracion.ucm.es/info/circulo/no25/rodriguez.pdf- 


\section{APENDICE}

Con la finalidad de mostrar algunos de los frutos obtenidos por estas investigadoras y compartir esta experiencia con docentes tanto en servicio como con los futuros docentes de español, se presenta información sobre el uso de regionalismos, utilizados por estudiantes de esa comunidad.

\section{Matriz 17}

Regionalismos utilizados por los estudiantes

\begin{tabular}{|l|c|c|}
\hline \multicolumn{1}{|c|}{ Regionalismos } & $\begin{array}{c}\text { Estudiantes de la 7-3 } \\
\text { que lo utilizan }\end{array}$ & $\begin{array}{c}\text { Estudiantes de la 8-1 } \\
\text { que lo utilizan }\end{array}$ \\
\hline "Rayé el examen". & 11 & 7 \\
"Seas boli”. & 9 & 6 \\
¿Dónde vive pa ir a dejarlo?” & 5 & 2 \\
¿Cómo le dicen?” & 14 & 9 \\
“Está enviajado". & 3 & 1 \\
“Qué buen chuzo" & 6 & 5 \\
\hline
\end{tabular}

Fuente: elaboración propia con base en el cuestionario a estudiantes, pregunta 8 .

El lector podrá observar que parte de las expresiones mencionadas por los alumnos encuestados están relacionadas con la zona geográfica a la que pertenecen y a ciertas actividades o labores propias de la zona.

Véase este otro ejemplo:

\section{Matriz 20}

\section{Palabras y frases que los estudiantes manejan} de forma diferente en su expresión oral

\begin{tabular}{|c|c|}
\hline Palabras o frases & Significados \\
\hline Pega hueco & $\begin{array}{l}\text { Que hizo algo mal } \\
\text { Ridículo } \\
\text { Una persona que pasa alguna vergüenza a cada rato }\end{array}$ \\
\hline Bañazo & $\begin{array}{l}\text { Pasar alguna vergüenza } \\
\text { ¿Qué ridículo! } \\
\text { Persona que hace tonterías } \\
\text { Polo }\end{array}$ \\
\hline $\begin{array}{l}\text { ¿dónde vive pa ir a dejalo? } \\
\text { Aseado }\end{array}$ & $\begin{array}{l}\text { Está loco } \\
\text { Mentiroso } \\
\text { Sin gracia } \\
\text { ¿qué le pasa? } \\
\text { Cochino } \\
\text { Inventor } \\
\text { No invente }\end{array}$ \\
\hline
\end{tabular}




\begin{tabular}{|l|l|}
\hline \multicolumn{1}{|c|}{ Palabras o frases } & \\
\hline ¿Cómo le dicen? & $\begin{array}{l}\text { Payaso } \\
\text { ¿Qué le pasa? } \\
\text { Sobretodo! }\end{array}$ \\
\hline Está enviajado & $\begin{array}{l}\text { Ilusionado } \\
\text { Está pijiado } \\
\text { Está en otro mundo }\end{array}$ \\
\hline Cachete explotao & $\begin{array}{l}\text { Es un saludo } \\
\text { Algo muy bueno (iSolo bueno!) } \\
\text { Me siento bien }\end{array}$ \\
\hline Está camote & $\begin{array}{l}\text { ¿Está loco! } \\
\text { Está bien } \\
\text { Está guapo } \\
\text { ¡Qué monte el suyo! } \\
\text { Lava huevos }\end{array}$ \\
\hline Échele moneda & $\begin{array}{l}\text { Se le ve el culo } \\
\text { Que le pague para que lo haga de nuevo }\end{array}$ \\
\hline Está solo & ¡nada que ver! \\
& $\begin{array}{l}\text { No invente } \\
\text { Baboso } \\
\text { Está guapísimo }\end{array}$ \\
\hline Frijolear & $\begin{array}{l}\text { Se fue de fiesta } \\
\text { Se fue de tonto }\end{array}$ \\
\hline Se fue de friquin & $\begin{array}{l}\text { Limpiar } \\
\text { Regañar }\end{array}$ \\
\hline Trapear & $\begin{array}{l}\text { Alguien muy alborotado } \\
\text { Alguien alegre } \\
\text { Bombas que se tiran en las fiestas }\end{array}$ \\
\hline Bombeta & $\begin{array}{l}\text { Robar } \\
\text { Descubrir }\end{array}$ \\
\hline Estafar \\
Enojar
\end{tabular}

Fuente: elaboración propia, con base en el cuestionario 2, aplicado a los estudiantes, pregunta 1.

Esta matriz es un ejemplo claro de la variedad lingüística que los alumnos de El Liceo del Roble de Heredia utilizan. En este caso, se torna fundamental aclarar que a pesar de que las personas pertenezcan a la misma comunidad no significa que el uso y manejo de su vocabulario sea el mismo. Cada individuo aprende también las variedades dentro de su grupo familiar o social, además, la influencia que ejerce el léxico de los padres es mucho más fuerte que el que aprenden por medio de sus amistades, profesores o compañeros, puesto que la mayor parte del tiempo la pasan con su familia. 
Según Lomas (1999), esta situación es una muestra de la riqueza léxica de cada uno de los estudiantes y de todos en su conjunto, así como su identidad personal, cultural y geográfica, por lo tanto, cada docente debe evitar cualquier tipo de prejuicio sobre la lengua de los alumnos y alumnas.

Ahora, veamos un ejemplo del significado que los docentes atribuyen a las palabras empleadas por sus alumnos.

\section{Matriz 21}

\section{Significado que atribuyen las docentes a} las palabras empleadas por sus estudiantes

\begin{tabular}{|l|l|l|}
\hline \multicolumn{1}{|c|}{ Palabra o frase } & \multicolumn{1}{|c|}{ Profesora 1 } & \multicolumn{1}{c|}{ Profesora 2 } \\
\hline Rayé el examen & Obtuve una nota bajo la & Me quedé en el examen \\
mínima & \\
Seas boli & N.R & No seas tonto \\
Dónde vive pa ir a dejarlo & N.R & N.R \\
Cómo le dicen & N.R & Está loco! \\
Está enviajado & Está drogado & Se drogó \\
Qué buen chuzo & Qué buen carro & Qué carrazo! \\
Qué pichudo & Qué lindo & Qué carga está! \\
Está operado & N.R & Está loco usted \\
Bañazo & Qué vergüenza & Pasar un ridículo \\
Pega hueco & N.R & N.R \\
Aseado & N.R & N.R \\
Está pijiado & Desconectado de la realidad & En otro mundo \\
Está camote & N.R & N.R \\
Cachete explotao & N.R & N.R \\
Cardetonta & Persona humilde, sencilla & Ingenua \\
Échele moneda & N.R & Vuelva a decirlo \\
Aquí es como en el cine & N.R & N.R \\
Está solo & Es único, diferente a los demás & Es inigualable \\
Paque & Para qué & Copiado \\
Mamé el examen & Perdí el examen, salí mal, & Me quedé en el examen \\
& no contesté todo & \\
Todo tour & N.R & Todo bien \\
Pura tusa & N.R & Pura paja \\
Chozón & Casa de lujo & Casa muy bonita y grande \\
Seas picha & N.R & No seas majadero \\
Pichudo & Bonito & Muy bonito \\
Tostado de la jupa & N.R & Loco \\
Está soñado & Está lindísimo & Algo muy bonito \\
Se fue de friquin & N.R & N.R \\
Qué legal & Qué honesto! & Está bien \\
Juma & Borracho & Borracho \\
\hline
\end{tabular}




\begin{tabular}{|l|l|l|}
\hline \multicolumn{1}{|c|}{ Palabra o frase } & \multicolumn{1}{|c|}{ Profesora 1 } & \multicolumn{1}{c|}{ Profesora 2 } \\
\hline Jartar & Comer & $\begin{array}{l}\text { Comer comida o hablar de } \\
\text { las personas }\end{array}$ \\
Trapear & Regañar & Regañar \\
Paquetear & Engañar & Plagiar \\
Bombeta & N.R & Alguien que es muy payaso, \\
& & o tiene muchas salidas \\
Cachar & Robar & Quitar algo sin permiso \\
Frijolear & N.R & N.R \\
\hline
\end{tabular}

Fuente: elaboración propia, con base en el cuestionario aplicado a docentes, pregunta única

En la matriz anterior es posible que el lector observe el conocimiento que poseen las docentes del vocabulario empleado por los estudiantes, a la vez, el significado que las docentes les atribuyen a esos conceptos. Según Arguedas y Ureña (2010), a pesar de que parezca sencillo identificar el lenguaje utilizado por los discentes, parece que ambas docentes no conocen en su totalidad el significado de las palabras que utilizan los jóvenes en sus clases, con sus amigos, o bien, en los recreos. Es posible que el motivo de esta situación esté relacionada con la brecha generacional entre docentes-alumnos- que no se manejen muchos de los términos que para los jóvenes son comunes.

Un docente de Español debe interesarse más por aprender sobre variedades lingüísticas, y a la vez, por el uso que los discentes le dan a la lengua, e instruir a los jóvenes en cuanto al uso adecuado de la lengua según la situación comunicativa presente, conocer el significado de la palabras que emplean para poder explicarles la importancia de sustituirlo por otro sinónimo, si la situación lo amerita, o bien, simplemente conocer el significado de esas palabras para ampliar su repertorio léxico y comprender mejor a sus estudiantes y así, contribuir con su formación académica y lingüística.

Avedaño y Miretti (2006), rescatan la importancia de que los docentes contribuyan con los estudiantes y les propicien situaciones de comunicación adecuadas, que a la vez, se potencie y reflexione sobre el uso de la lengua oral.

Es importante que los profesores procuren comprender el sentido de las diferentes actividades comunicativas, sus propósitos, sus contenidos y su estructura para dar cuenta del tipo de interacciones comunicativas que ocurren en cada una de ellas y el tipo de discurso que se genera, así podría comprenderse que el uso de regionalismos es una riqueza léxica y no considerarlo como un vicio del lenguaje. 